\title{
La sicurezza del paziente in Medicina di Laboratorio. La "Grande Visione" dieci anni dopo
}

\author{
Patient Safety in Laboratory Medicine. The Big Picture ten years later
}

\author{
Piero Cappelletti ${ }^{1}$ (D)
}

Ricevuto: 25 dicembre 2017 / Accettato: 22 gennaio 2018 / Pubblicato online: 5 febbraio 2018

(C) Società Italiana di Patologia Clinica e Medicina di Laboratorio 2018

Riassunto Dieci anni fa fu presentata una Grande Visione (the Big Picture) intorno alla sicurezza del paziente (Patient Safety) in Medicina di Laboratorio, costituita dai cerchi concentrici della sicurezza dei risultati (qualità analitica), del processo (Total Testing Process, TTP), degli esiti (efficacia clinica e appropriatezza) e della sicurezza del cittadino. Da allora le innovazioni più importanti al livello della sicurezza dei risultati sono state l'introduzione di nuovi strumenti di controllo analitico (Individualized Quality Control Plan, IQCP) e di accreditamento (ISO 15189:2012) e la ripresa della discussione teorica (criteri di definizione della prestazione analitica; dibattito tra Total Analytical Error-TAE e Measurement Uncertainty-MU come misure dell'accuratezza) soprattutto grazie a EFLM (European Federation of Clinical Chemistry and laboratory Medicine). Tuttavia i dati riportati da Westgard et al nel 2017 mostrano ancora livelli di accuratezza analitica inferiori a 3 sigma, un uso non corretto dei controlli di qualità interni in oltre il $50 \%$ dei casi, uno scarso utilizzo di IQCP in termini di laboratori $(<20 \%)$ e di test $(<5 \%)$, un impatto clinico pressoché nullo di MU $(<5 \%)$. Il tema dell' affidabilità analitica dei risultati di laboratorio, dunque, è ancora essenziale in Medicina di Laboratorio.

Per quanto riguarda il TTP, tre sono i percorsi più rilevanti che in questi anni ne hanno potenziato il significato: lo studio e l'identificazione di indicatori di qualità (Quality Indicators, QI) per le fasi extra-analitiche, in assonanza con i requisiti ISO 15189:2012 e inseriti in un quadro di risk management che ne consente benchmark, analisi delle cause di fallimento e azioni correttive; la pubblicazione da parte di The National Academy of Science del documento Impro-

$\varangle$ P. Cappelletti

pie.cappelletti@gmail.com

1 SIPMeL, Castelfranco Veneto, TV, Italia ving Diagnosis in Health Care che rappresenta un cambio di paradigma, perché si passa dall'attenzione alla riduzione degli errori all'impegno per il miglioramento della diagnosi, con definizione dell' errore diagnostico dal punto di vista del paziente e del processo diagnostico come un lavoro dinamico e collaborativo di gruppo; la revisione del brain-tobrain loop di Lundberg, con la fusione con il concetto di interfaccia clinica-laboratorio amplificata, la definizione delle fasi pre-pre e post-post analitiche come momenti di condivisione clinici-laboratoristi, il concetto di colloquio tra reti di cervelli e la centralità del paziente e dei suoi esiti.

Dal punto di vista degli esiti, le tesi più interessanti poggiano sul concetto di "utilità clinica" dei test di laboratorio che esprime il passaggio da una visione "essenzialista" a una "consequenzialista" della Medicina di Laboratorio. Tuttavia restano i problemi della complessità degli esiti clinici in senso lato, la sovrapposizione tra efficacia clinica e appropriatezza e la difficoltà di evidenze che correlino esplicitamente test ed esito clinico. Le evidenze vengono sintetizzate in linee guida che diventano il criterio di valutazione di appropriatezza ed efficacia. Da questo punto di vista le linee guida cliniche e quelle di laboratorio necessitano di esplicite raccomandazioni per tutte le fasi del TTP, che solo i professionisti della disciplina sono in grado di produrre. Infine il ruolo sociale della Medicina di Laboratorio nella sicurezza del cittadino è enfatizzato in tempi di sfiducia nella scienza e di proliferare di informazioni false e tendenziose. Il compito dei Laboratori nell'informazione/formazione dell'opinione pubblica ne è amplificato.

Alla luce di quanto sopra, la Grande Visione è ancor più necessaria per la sicurezza dei pazienti e dei cittadini e per la promozione della salute.

Parole chiave Sicurezza del paziente $\cdot$ Affidabilità dell'esame - Total Testing Process · Efficacia clinica · Appropriatezza $\cdot$ Utilità clinica $\cdot$ Medicina di Laboratorio 
Summary Ten years ago a Big Picture on Patient Safety in Laboratory Medicine was presented as a figure with four concentric circles representing all deficiencies in the quality of laboratory testing: analytical results, total testing process (TTP) quality, outcomes safety as appropriateness and effectiveness, and citizens' safety. Since then, several innovations have been presented in every circle of the Big Picture.

For analytical accuracy, new tools for internal quality control (IQC) as Individualized Quality Control Plan (IQCP) by CLSI and for accreditation as ISO 15189:2012 were introduced; the quality control rules for new tests and methods as genetic tests or Next Generation Sequencing (NGS) were established; a review of criteria for the analytical performance and for assessing analytical methods was carried out by European Federation of Clinical Chemistry and Laboratory Medicine (EFLM). Nevertheless, Westgard et al recently reported that in US surveys several tests show an analytical performance $<3$ sigma; the majority of laboratories use IQC in an incorrect way; few laboratories use IQCP and for few test; few laboratories report the Measurement Uncertainty (MU), with very limited effect on the clinical diagnostic process. Then, analytical accuracy is still now a major issue in the practice of laboratory medicine.

TTP is a well-known concept and three new visions enriched it in ten past years. Quality indicators for every phase of TTP were studied, defined, quantified and used in a risk assessment framework for benchmark, root analysis, and corrective actions. The "Improving Diagnosis in Health Care", a report by The National Academy of Science, represented a paradigm shift: the focus moves from the diagnostic error reduction to the goal of improving diagnosis; diagnostic error is defined from the patient point of view; the diagnostic process is a dynamic, complex, iterative, and collaborative teamwork. A new vision of the Brain-to-Brain loop was presented, with specifications about the fusion with the clinic-laboratory interface concept, the loop as a conversation between networks of brains, a new definition of pre-pre and post-post analytical phases, and the centeredness of the patient and his outcomes.

The attention for "clinical utility" became central in the debate about outcomes in Laboratory Medicine, as a part of a conceptual shift from an essentialist vision of Laboratory Medicine to a consequentialist one. This conceptual evolution pointed out three main issues: the complexity of patients' outcomes; the overlapping of concepts of appropriateness and effectiveness; the difficulties about methods and tools for demonstrating the connection between tests and outcomes. Nevertheless, guidelines, as synthesis of evidences, became the criteria for assessing appropriateness and evaluating clinical efficacy. Clinical guidelines very often lack of information about the characteristics of tests and their TTPs. Professional bodies should be very active in this field.
Finally, the need for information and education of the public about science, medicine, laboratory medicine, and tests defined the "social" role of Laboratory Medicine Societies. A Big Picture, as described above, is still now useful and necessary as framework for a comprehensive Patient Safety in Laboratory Medicine.

Keywords Patient safety $\cdot$ Test accuracy . Total testing process - Effectiveness - Appropriateness - Clinical utility . Laboratory Medicine

\section{Sicurezza del paziente: la Grande Visione}

Steven Woolf, epidemiologo americano specialista in medicina preventiva e salute pubblica e membro dell'US Preventive Service Task Force e dell'Institute of Medicine, scrive nell' articolo "Patient Safety is not enough: targeting quality improvements to optimize the health of the population" [1] che la sicurezza del paziente (patient safety) è certamente importante per migliorare la qualità delle cure, ma è solo un aspetto che va integrato in una visione globale (the Big Picture), un'immagine di quattro cerchi concentrici dove il più interno rappresenta gli errori nella sicurezza del paziente (rischi aggiuntivi dipendenti da violazioni, errori e mancanze dei curanti); il secondo, che ricomprende il primo, è quello degli errori medici (non solo i rischi attivi ma anche quelli derivanti da omissioni del miglior percorso preventivo); il terzo ancora più esterno degli errori nella qualità (cure che non raggiungono gli standard e gli esiti definiti, non solo per fattori individuali ma per errori di disegno e di organizzazione); infine il quarto più esterno degli errori del "prendersi cura" (cure insufficienti rispetto ai benchmark ma anche rispetto ai bisogni e alle aspettative dei pazienti).

Parafrasando il concetto di "Big Picture" di Steven Wolff, anche la sicurezza del paziente in Medicina di Laboratorio è stata rappresentata [2], in una visione globale, come una serie di cerchi concentrici-sicurezza del risultato (qualità analitica), sicurezza del processo (qualità del total testing process), sicurezza dell'esito (effectiveness e appropriatezza), sicurezza del cittadino (ruolo sociale della Medicina di Laboratorio)—al Congresso Nazionale SIMeL del 2006 dedicato alla "Sicurezza del Paziente"-considerato come il completamento dell'analisi a tutto tondo della Medicina di Laboratorio iniziata con i temi organizzativi, più cogenti, a Modena nel 2004 e proseguita con il fulcro disciplinare individuato nella risposta al quesito clinico a Trieste nel 2005-e al Forum Risk Management in Sanità ad Arezzo nel 2007.

A dieci anni di distanza, il concetto della Grande Visione riguardo al tema sicurezza/qualità in Medicina di Laboratorio è ancora valido? 


\section{Sicurezza del risultato analitico}

Dieci anni fa il tema dell'attenzione al problema analitico, del posizionamento del dato analitico nel quadro complessivo dell'incertezza della misura, della comunicazione adeguata di essa erano ancora frontiere armate e punti focali della battaglia per la sicurezza del paziente [3]. Ed erano [4] e sono [5] ben note le conseguenze sulla mis-classificazione diagnostica dei pazienti dell'inaccuratezza delle misure, in particolare in alcune aree (TDM, monitoraggio degli anticoagulanti, $\mathrm{HbA}_{1 \mathrm{c}}$, test genetici). Tuttavia, il progresso tecnologico ha diffuso una convinzione di "certezza" della misura, che coinvolge clinici e laboratoristi, basata sulla perfezione tecnica dei device/strumenti e Linda Thienpont [6] si chiedeva chi avrebbe baciato la Bella Addormentata (l'accuratezza), sostituita molto spesso nell'automazione dalla ripetibilità e dalla semplificazione e nelle analisi decentrate dalla semplicità e dall'immediatezza. Sottolineava, inoltre, come questo non potesse essere un compito esclusivo della professione, ma dovesse coinvolgere in un rapporto dialettico anche l'industria che sviluppa e appronta i test. In questi dieci anni l'attenzione per la qualità analitica e la sicurezza dei risultati è stata tenuta alta, sia sotto il profilo degli strumenti offerti per il suo controllo sia sotto il profilo teorico.

Sotto il profilo pratico, CLSI EP23 [7] traduce i principi della gestione del rischio industriale (risk management) nella pratica del laboratorio clinico a supporto dei sistemi di gestione della qualità, che si sono dimostrati efficaci (ISO 9001 e 15189) nel promuovere la sicurezza del paziente [8]. EP23 tiene conto della varietà e del livello dei sistemi di controllo incorporati nelle nuove strumentazioni (sia per monitorarne automaticamente il funzionamento, sia per integrare i classici controlli di qualità interni), ma anche della verifica scientifica dell'effettivo rischio di errore nelle diverse fasi del processo analitico (risk assessment), per definire un "piano di controllo" che integri i diversi modi di verifica della qualità in forma specifica, monitorata e costantemente rivalutata, e "su misura" (Individualized Quality Control Plan, IQCP) [9].

ISO 15189:2012 [10] suggerisce di sostituire come criterio per la performance analitica all'Errore Totale Analitico (Total Analytical Error, TAE) e all'Errore Totale Ammissibile (Allowable Total Error, ATE) l'Incertezza della Misura (Measurement Uncertainty, MU), proposta antica ma mai effettivamente perseguita per le difficoltà di eliminare il bias nella valutazione dell' accuratezza. La proposta non è stata accettata da CLIA (Clinical Laboratory Improvement Amendments) ed è stata criticata, dando origine a un serrato dibattito sulla rivista Clinical Chemistry and Laboratory Medicine [11-13].

L'European Federation of Clinical Chemistry and Laboratory Medicine (EFLM), in particolare, nella conferenza del 2014 "Defining analytical performance goals 15 years after the Stockholm Conference on Quality Specifications in Laboratory Medicine" [14] rivede la gerarchia per definire la qualità della prestazione analitica, semplificando il quadro del 1999 con l'eliminazione dei criteri suggeriti dai corpi professionali o istituzionali (organismi di controllo e organizzatori di VEQ) e stabilendo un ordine basato sugli esiti clinici, diretti e indiretti, sulla variabilità biologica e sullo stato dell'arte [15], e ripropone l'uso di MU, anche per adeguarsi alle indicazioni di ISO 15189:2012. Nel 2015, EFLM tiene un corso intitolato "How to assess the quality of your method?" [16] nel quale affronta le specifiche di qualità, la variabilità biologica, la misura dell'incertezza, la verifica del bias e dell'imprecisione, il rapporto tra $\mathrm{LoB} / \mathrm{LoD} / \mathrm{LoQ}$ e livelli decisionali, la comparabilità di metodi e strumenti, gli intervalli di riferimento, la verifica delle procedure qualitative, i controlli di qualità interni ed esterni e l'uso della metrica Six Sigma. In Italia, la Commissione Qualità di SIPMeL rilascia un importante documento sulla comparabilità di metodi e strumenti [17], uno dei punti più delicati della qualità analitica.

Lo stretto legame tra qualità analitica e qualità clinica è ora enfatizzato, per esempio in relazione ai percorsi diagnostico-terapeutici-assistenziali (PDTA) [18]. La scelta dei metodi (iPTH, CRP al posto di VES) e degli strumenti (POCT per $\mathrm{HbA}_{1 \mathrm{c}}$, ACR, PTT/INR, HIV e TBC) e la loro verifica di qualità, così come la scelta dei biomarcatori (PNC; hs-cTn vs cTn) e dei loro limiti decisionali e delle sequenze organizzative (esami reflex o riflessivi), sono elementi decisivi per il funzionamento dei PDTA. La necessità di un' affidabilità analitica perfetta è, d'altro canto, affrontata dall'emergere di esami di laboratorio di elevatissimo impatto clinico e sociale ma dal processo analitico assai indaginoso, come i test molecolari o di Next Generation Sequencing (NGS), per i quali sono stati messi a punto strumenti di validazione e controllo adeguati $[19,20]$.

Tuttavia, quest'anno James Westgard e altri pubblicano il libro "Risk, Error and Uncertainty: Laboratory Quality Management in the Age of Metrology" [21], nel quale affrontano, tra l'altro, i temi metrologici e della tracciabilità analitica, il dibattito tra Errore Totale Analitico (TAE) e Incertezza della Misura (MU), la variabilità biologica, lo stato dell' arte nella definizione degli obiettivi, la comparabilità dei risultati, i controlli statistici compreso IQCP e l'uso della metrica Six Sigma $(\sigma)$. L'aspetto preoccupante è la differenza tra le teorie raffinate, proposte a livello internazionale, e la realtà rilevata da indagini condotte da Westgard tra il 2014 e il 2017. La qualità analitica non è sempre buona, come dimostra l'indagine $2016 \mathrm{del}$ College of American Pathologists (CAP) su 3237 laboratori [22]. Nell'esame del campione di controllo per l' $\mathrm{HbA}_{1 \mathrm{c}}$, per esempio, solo un sottogruppo metodologico su 24 ha superato i $3 \sigma$, mentre 8 si sono piazzati tra 3 e 2 sigma e 12 sotto i $2 \sigma$. Si ricordi che l'obiettivo dovrebbe essere $6 \sigma$, una qualità media potrebbe essere $4 \sigma$ e la qualità minima in campo industriale è $3 \sigma$. 
Benché l'analita sia particolarmente difficile per complessità fisico-chimiche, caratteristiche della matrice, tracciabilità ed eterogeneità di metodi, il risultato è sconfortante pensando all'impatto sulla diagnosi e sul monitoraggio della malattia diabetica. I controlli di qualità interni (CQI) non sono gestiti al meglio [23]: il 55\% dei laboratori usa regole superate e il 62,5\% limiti troppo larghi; il 64\% utilizza il controllo del produttore, il 51,5\% lo analizza solo 1 volta al giorno e il 49,5\% solo all'inizio del lavoro; 1 su 3 va fuori controllo ogni giorno. Dall'indagine emerge anche una percezione della propria qualità non realistica: 1 su 3 ha una valutazione ottimistica del numero di campioni rifiutati ogni giorno $(<1 \%)$ e 1 su 10 pensa di emettere i risultati a prescindere dal CQI. Per quanto riguarda IQCP, in una specifica survey americana [24], solo 1 su 5 dei rispondenti lo applica e solo per una piccola percentuale di esami $(<5 \%)$; nell'analisi del rischio propedeutica, nel $68,7 \%$ dei casi non rileva rischi inaccettabili e nel 79,3\% pensa di essere comunque in condizioni di sicurezza, a prescindere dall'uso di IQCP. Desta perplessità la diversità di risultati nell'applicazione dei metodi di valutazione del rischio: in un percorso POCT dove gli esperti rilevano 91 punti di rischio i laboratori americani ne mettono in evidenza 30 e quelli non americani partecipanti all'indagine solo 10. Questi dati sollevano dubbi sulla riproducibilità dei metodi utilizzati [25]. Infine, il 68,7\% di chi utilizza IQCP effettua controlli di qualità 1 volta al mese. Conclude amaramente Westgard che IQCP sembra "un esercizio cartaceo, allo scopo di giustificare una frequenza di controlli interni già ridotta, dando a questo taglio una parvenza scientifica". Infine, in un' altra indagine [26], solo una piccola parte dei rispondenti americani utilizza MU (15\%) e/o la include nelle risposte $(7,5 \%)$, le motivazioni sono legate all'applicazione di ISO 15189 e l'effetto è trascurabile, soprattutto in relazione a modifiche delle decisioni cliniche $(<5 \%)$. Il tema dell'accuratezza analitica e dei suoi metodi di valutazione e controllo è ancora del tutto aperto.

\section{Sicurezza dei processi in Medicina di Laboratorio}

L'importanza fondamentale del controllo dell'intero processo dell'esame di laboratorio (il total testing process come descritto magistralmente da Lundberg [27]) e non solo della fase analitica per un risultato affidabile per il clinico e il paziente era già chiaro dieci anni fa. Da questo punto di vista vi sono stati almeno tre percorsi di grande rilevanza.

Sulla scorta dei propri lavori sull'errore in medicina di laboratorio [28], che avevano mostrato la prevalenza della fase pre- e post-analitica nel determinare l'errore cosiddetto di laboratorio, e dei primi tentativi di Ricos et al [29], Mario Plebani, anche all'interno della EFLM Task Force on Performance Specifications for the extra-analytical phases, si è votato allo studio e alla proposizione di una serie di indicatori di qualità (QI) [30] in grado di monitorare adeguatamente tutti i passaggi delle fasi pre- e post-analitiche, oltre che di quella analitica, in relazione alle indicazioni di ISO $15189 \mathrm{e}$ con l'individuazione degli obiettivi di difettosità ammissibile (mis-identificazione del paziente; inappropriata richiesta; qualità formale della richiesta; qualità del campione; tempo della raccolta/prelievo; qualità analitica come CQI e VEQ; TAT; qualità formale della risposta; qualità dei risultati; notifica degli stessi; commenti interpretativi; esiti). Infine il complesso degli indicatori di qualità è inserito in un quadro di riferimento, basato sul risk management, che consente benchmark, analisi delle cause e azioni correttive [31].

Nel dicembre del 2015 The National Academy of Scien$c e$ (che ha incorporato Institute of Medicine, IOM) pubblica un rivoluzionario documento dal titolo Improving diagnosis in Health Care [32], che è certamente l'evoluzione dei fondamentali lavori dello IOM sugli errori in Medicina (To err is human, 2000 e Crossing the quality chasm, 2001), ma definisce un cambiamento di paradigma, perché si passa dall'attenzione alla riduzione degli errori all'impegno per il miglioramento della diagnosi. Tre sono i temi principali affrontati dal documento: il focus è sull'errore diagnostico, che finora aveva avuto minore attenzione per diverse ragioni (dati dispersi, poche misure affidabili, analisi prevalentemente retrospettive); l'errore diagnostico è definito dal punto di vista del paziente; il processo diagnostico è complesso, iterativo e collaborativo (team intra e interprofessionali). L'errore diagnostico è definito come "il fallimento in: (a) stabilire una spiegazione accurata e tempestiva dei problemi di salute del paziente o (b) comunicare tale spiegazione al paziente stesso". La centralità del paziente è ben enfatizzata, con i corollari della partnership e del coinvolgimento delle famiglie (intese come persone che si prendono cura). Il lavoro diagnostico è un processo dinamico di gruppo che condivide obiettivi, ha chiari i ruoli, mutua fiducia, efficace comunicazione e misura processi ed esiti. I determinanti del lavoro collaborativo sono la centralità del paziente, l'impegno della leadership, le caratteristiche della comunicazione, la consapevolezza delle identità e interdipendenze, la responsabilità e una finalizzata struttura dell'organizzazione. Risuona qui la concordanza con i concetti societari dell'equipe di laboratorio. Altri punti, infine, vengono sottolineati ed entrano nelle Raccomandazioni: l'importanza dell'apprendimento continuo sulla base della "learning science", la centralità della health information technology, l'importanza del disegno e dell' organizzazione del lavoro e la fondazione di una cultura conseguente.

Il concetto di total testing process e di brain to brain loop è stato rivisto più volte negli anni [33,34]. Nel 2017, riprendendo l'elaborazione precedente del 2007 e 2010, ho proposto una nuova visione [35] del ciclo di Lundberg per renderlo pienamente utilizzabile anche oggi e nel prossimo futuro, anche per gli esami fuori dal laboratorio (POCT) e dall'ospedale (territorio, home), come schema teorico-pratico della generazione dell'informazione medica di laboratorio. Le 
condizioni necessarie sono: (1) lo schema sia fuso con il concetto di interfaccia clinica-laboratorio di Büttner, amplificata dall'organizzazione a rete del laboratorio fuori dalle mura; (2) le fasi pre-pre e post-post siano pensate non solo come la parte extra-lab delle fasi pre e post analitiche ma come momento più alto del lavoro all'interfaccia (team multidisciplinari per "evidenze", linee guida, protocolli di appropriatezza, feedback e audit); (3) venga descritto come colloquio tra reti di cervelli (Laboratorio-team clinico e assistenziale-paziente); (4) il paziente sia effettivamente al centro del ciclo di generazione dell'informazione medica a lui dedicata (problema di salute-negoziazione su valori e preferenze- effectiveness). Come si vede, alcuni concetti maturano di pari passo con altre riflessioni precedentemente riportate (il lavoro a team, la centralità del paziente), ma altre sono del tutto originali (l'interfaccia amplificata e il lavoro delle fasi pre-pre e post-post mai così definite prima d'ora). Questa teorizzazione, sufficientemente descrittiva seppur sintetica, può essere assunta come riferimento per mostrare i luoghi, metaforici e non, in cui si manifestano i ruoli cui sono chiamati oggi i professionisti della Medicina di Laboratorio (sacerdoti della tecnologia, pianificatori e manager, consulenti clinici e attori del governo clinico) e come modello per l'identificazione dei punti critici delle attività correlate all'obiettivo qualità/assenza di errori/sicurezza del paziente.

\section{Sicurezza degli esiti}

Il tema degli esiti in medicina è divenuto centrale in quest'ultima decade, come evoluzione dell'outcome movement iniziato alla fine degli anni ' 80 in America. Dal punto di vista del Laboratorio le considerazioni probabilmente più interessanti stanno all'interno di una più matura considerazione del valore della Medicina di Laboratorio, che muove dalla valutazione "essenzialista" puntata sul turnover dei risultati (accuratezza e validità) a una "consequenzalista" puntata sul valore delle conseguenze (utilità ed esiti di salute), dalla parola d'ordine "verità" a quella di "utilità" [36]. Nonostante il ruolo fondamentale del concetto di accuratezza diagnostica, vi è una spinta crescente ad avere informazioni sui test che trascendano la prestazione tecnica e analitica per attingere a una più ampia prospettiva che riferisce agli esiti del paziente. In questo senso viene introdotto e specificato il concetto di utilità clinica (informazioni utili agli esiti), talora correlato all'apprezzamento dei clinici (diagnostic thinking effcacy) o all'effetto sul trattamento (therapeutic efficacy), ma meglio descritto come dipendente dagli esiti clinici, primari e secondari, del paziente (patient outcome efficacy). Bossuyt et al [36] utilizza il termine "utilità" piuttosto che "efficacia", perché vuole rilevare come le conseguenze delle decisioni cliniche basate sul test debbano essere esplicite e quantificate. L'aggettivo "clinica" ha a che fare con gli esiti di salute individuale e collettiva, comprende non solo le conseguenze dirette del test ma anche quelle derivate dalle strategie di intervento ad esso conseguenti, indica il valore probabilistico (non necessariamente tutti gli esiti sono presenti in ciascun paziente) e comparativo (il riferimento è la miglior pratica standard) della utilità.

Questi concetti pongono alcuni problemi [37]: la definizione degli esiti "clinici" in senso lato; la sovrapposizione dei concetti di "effectiveness" e "appropriateness"; la difficoltà di produrre "evidenze" del legame test/outcome. Bossuyt definisce come esiti da misurare, accanto a quelli primari legati alla decision making e all'effetto di trattamento/attesa, quelli secondari (emotivi, sociali, cognitivi, comportamentali). La visione si collega alla progressiva centralità del paziente, in senso olistico, nella Medicina occidentale, ma pone grandi problemi metodologici. Nonostante la rimarcata caratteristica "probabilistica" del consequenzialismo, se il termine di valutazione sono gli outcome dei pazienti è difficile non vedere come i concetti di efficacia clinica e di appropriatezza si sovrappongano, anche se guardano l'una più alla parte della selezione diagnostica, l'altra alla parte interventistica. La complessità del termine appropriatezza è stata recentemente illustrata [38]. Tuttavia, l'insieme delle visioni descritte in precedenza, e in particolare quella sostenuta in Improving Diagnosis in Health Care [32], convergono verso l'idea che i processi produttivi in medicina siano complessi, iterativi e collaborativi. In Medicina di Laboratorio, è evidente che l'azione lungo tutto il TTP dell'equipe di laboratorio con la sottolineatura del nuovo concetto di fasi pre-pre e post-post, è la condizione sia dell'appropriatezza sia degli esiti [38].

Il punto della produzione delle evidenze è particolarmente delicato ed è stato più volte esaminato $[37,39]$. Nella difficoltà di valutare direttamente gli esiti, il metro di giudizio si è spostato sull'aderenza alle linee guida, sintesi delle evidenze disponibili [40]. Pur nella consapevolezza che si tratta di una scorciatoia, questo sistema è accettato universalmente, ma le difficoltà nella produzione di linee guida sono state più volte sottolineate [41]. La produzione di linee guida di Medicina di Laboratorio (LGML) ha poi specifiche problematiche [42]. Principalmente, da un lato, la difficoltà di correlare l'utilizzo di esami di laboratorio agli esiti clinici del paziente e quindi di avere studi primari di alta qualità, come sono per definizione i trial clinici randomizzati (RCT), sposta l'attenzione verso esiti surrogati e/o l'accuratezza diagnostica, peraltro non sempre facilmente perseguibili. Di qui l'ampio utilizzo di esperienze e pareri di esperti. Dall'altro, nella pratica del laboratorio sono spesso gli aspetti tecnologici e organizzativi che hanno la necessità di essere indirizzati (guidance), soprattutto quando questi aspetti, che possono apparire "interni" rispetto al lavoro all'interfaccia clinica-laboratorio, condizionano invece pesantemente il percorso diagnostico e quindi terapeutico del paziente. 
Infine è noto [43] come le linee guida cliniche abbiano generalmente una bassa considerazione per gli aspetti considerati laboratoristici, rispettando in media solo in un terzo dei casi le necessarie informazioni riguardo lo stato dei pazienti, le interferenze biologiche e analitiche e il trattamento dei campioni, e che l'inserimento di uno specialista di laboratorio nei comitati/gruppi di preparazione di line guida cliniche migliora la situazione rendendone circa la metà esaustive sotto il profilo dei mezzi diagnostici. La necessità della presenza dei laboratoristi nei gruppi di produzione, adozione, adattamento e validazione delle linee guida deve essere ben presente sia a livello professionale sia a livello istituzionale. SIPMeL ha definito un chiaro documento a sostegno di obiettivi e metodi per le linee guida e le LGML [42].

\section{Sicurezza del cittadino}

Si è visto, anche in questo articolo e a più riprese, come il tema della centralità del paziente [44] sia presente nelle verifiche degli errori ai diversi livelli e nel concetto più ampio di miglioramento della qualità, in questo caso diagnostica. $\mathrm{Nel} 2006$ un'approfondita indagine su "Diritti dei cittadini e laboratorio analisi" [45] esamina il rispetto dei quattordici diritti fondamentali della "Carta europea dei diritti del malato" approvata nel 2002 (misure preventive, accesso, informazione, consenso, libera scelta, tempo del paziente, privacy e confidenzialità, standard di qualità, sicurezza, innovazione, evitare sofferenze, trattamento personalizzato, reclamo, risarcimento). Nel 2016 i diritti dei pazienti vengono declinati secondo i passaggi del TTP [46]. Ma la sicurezza del cittadino, nella nostra visione, non si riduce all'affermazione, seppure importante, di diritti individuali e il compito della Società non è solo di garanzia di accesso. Il tema, infatti, non è solo rivolto al paziente ma al complesso della società, ai cittadini sia in termini di specifica prevenzione delle malattie sia in termini di educazione complessiva sui temi della malattia, della salute e della scienza. Questo, che abbiamo definito "ruolo sociale della Medicina di Laboratorio", è stato ben presente in tutte le attività societarie, da quelle organizzative interne [47] a quelle legate all'innovazione [48] e a quelle strettamente legate ai temi della sicurezza/qualità [2]. Il compito di informazione/formazione si è oggi esteso, a fronte del dilagare delle fake news e del rifiuto della scienza [49]: comunicare la scienza; spiegare il significato dei test e del processo diagnostico; far comprendere il concetto di rischio e probabilità; assumere un ruolo critico nei confronti delle forzature scientifiche e mediatiche; promuovere la consapevolezza di "attenti alle bufale". Si tratta di un tema e di una sensibilità che ci ha contraddistinto, in Italia e nel mondo, ma non siamo soli. David Eagleman [50], neuroscienziato cognitivo del Baylor College of Medicine di Houston, ha definito l'impegno etico del "ruolo pubblico", invitando gli scienziati e i medici a rendicontare i benefici delle ricerche, senza trucchi o trionfalismi; a contribuire alla diffusione di una cultura scientifica, mettendo a disposizione le proprie specifiche abilità; a contrastare la disinformazione; a sporcarsi le mani con la politica, in termini legislativi; a spiegare cos'è la scienza (riproducibilità e metodo) e soprattutto cosa non è (la Verità); a educare alla consapevolezza dell'incertezza, del rischio e delle trappole mentali con cui abbiamo a che fare quando ne parliamo.

\section{La Grande Visione dieci anni dopo}

In questi ultimi dieci anni molte cose sono cambiate: nuovi e rinnovati metodi e teorie per l'accuratezza dei risultati, una completa accettazione del concetto di total testing process e del ruolo del Laboratorio nel processo diagnostico, il tentativo di valutare esplicitamente gli esiti della diagnostica, la consapevolezza del necessario impegno culturale oltre che tecnico-scientifico. Molte altre restano ancora da fare. È necessario migliorare la qualità analitica in un mondo che la dà per scontata; affrontare i temi divisivi sui criteri di valutazione e controllo dei test; diffondere il concetto di fasi pre-pre e post-post come momenti alti di scambio all'interfaccia; realizzare efficaci modelli di valutazione degli esiti legati alla diagnostica; definire linee guida che comprendano gli aspetti delicati preanalitici analitici e postanalitici che solo il Laboratorio conosce; rendersi visibili professionalmente e culturalmente a tutta la società. Penso, quindi, che lo schema della Grande Visione possa rappresentare ancora un modello cognitivo adeguato alla sistematizzazione della sicurezza del paziente nella Medicina di Laboratorio e che anzi risponda meglio di altri framework alle innovazioni avvenute e alle nuove sensibilità, in particolare con il documento Improving Diagnosis in Health Care, e che faciliti il passaggio del Laboratorio da servizio essenziale a determinante integrato degli esiti del paziente e nella promozione della salute.

\section{Conflitti di interesse Nessuno.}

Studi condotti su esseri umani e animali Per questo tipo di studio non è richiesto l'inserimento di alcuna dichiarazione relativa agli studi effettuati su esseri umani e animali.

\section{Bibliografia}

1. Woolf SH (2004) Patient safety is not enough: targeting quality improvements to optimize the health of the population. Ann Intern Med 140:33-36

2. Cappelletti P (2006) La Sicurezza del Paziente e la Medicina di Laboratorio. RIMeL-IJLaM 3(Suppl):17-35 
3. Westgard JO, Westgard SA (2006) The quality of laboratory testing today: an assessment of sigma metrics for analytic quality using performance data from proficiency testing surveys and the CLIA criteria for acceptable performance. Am J Clin Pathol 125:343-354

4. Klee GG, Schryver PG, Kisabeth RM (1999) Analytic bias specifications based on the analysis of effects on performance of medical guidelines. Scand J Clin Lab Invest 59:509-512

5. Sikaris K (2015) Performance criteria of the post-analytical phase. Clin Chem Lab Med 53:949-958

6. Thienpont LM (2008) Accuracy in clinical chemistry-who will kiss sleeping beauty awake? Clin Chem Lab Med 46:1220-1222

7. CLSI (2011) EP23-A. Laboratory quality control based on risk management; Approved Guideline. CLSI document EP23-A Wayne, PA, USA:CLSI

8. Allen LC (2013) Role of a quality management system in improving patient safety-laboratory aspects. Clin Biochem 46:1187-1193

9. Drago C (2015) Qualità e sicurezza per il PoCT: controllo del processo secondo EP23-A del CLSI. Riv Ital Med Lab 11:55-60

10. ISO 15189 (2012) Medical laboratories-requirements for quality and competence. International Organization for Standardization, Geneve

11. Westgard JO (2016) Useful measures and models for analytical quality management in medical laboratories. Clin Chem Lab Med 54:223-233

12. Ooosterhuis WP, Theodorsson E (2016) Total error vs. measurement uncertainty: revolution or evolution? Clin Chem Lab Med 54:235-239

13. Panteghini M, Sandberg S (2016) Total error vs. measurement uncertainty: the match continues. Clin Chem Lab Med 54:195-196

14. Panteghini M, Sandberg S (2015) Defining analytical performance specifications 15 years after the Stockholm conference. Clin Chem Lab Med 53:829-832

15. Sandberg S, Fraser CG, Horvath AR et al (2015) Defining analytical performance specifications: consensus statement from the 1st strategic conference of the European federation of clinical chemistry and laboratory medicine. Clin Chem Lab Med 53:833-835

16. Topic E, Nikolac M, Panteghini M et al (2015) How to assess the quality of your analytical method? Clin Chem Lab Med 53:17071718

17. Pradella M, Cesana BM (2016) Linee guida per il confronto di procedure di esami di laboratorio: utilizzo delle indicazioni di CLSI EP09-A3 ed EP31-A-IR. Riv Ital Med Lab 12:26-35

18. Horvath AR, Bossuyt PM, Sandberg S et al (2015) Setting analytical performance specifications based on outcome studies-is it possible? Clin Chem Lab Med 53:841-848

19. Mattocks CJ, Morris MA, Matthijs G et al (2010) A standardized framework for the validation and verification of clinical molecular genetic tests. Eur J Hum Genet 18:1276-1288

20. Aziz N, Zhao Q, Bry L et al (2015) College of American Pathologists' laboratory standards for next-generation sequencing clinical tests. Arch Pathol Lab Med 139:481-493

21. Westgard JO, Armbruster D, Westgard SA (2017) Risk, error and uncertainty: laboratory quality management in the age of metrology, An Issue of the Clinics in Laboratory Medicine. Elsevier, Amsterdam

22. Westgard JO, Westgard SA (2017) Measuring analitycal quality: total analytical error versus measurement uncertainty. Clin Lab Med 37:1-13

23. https://www.westgard.com/great-global-qc-survey-results.htm (Accesso 25 novembre 2017)

24. https://www.westgard.com/downloads/other-downloads/72-2015iqcp-survey-chartpack/file.html (Accesso 25 novembre 2017)

25. Shelb NA, Franklin BD, Barber N (2009) Is failure mode and effect analysis reliable? J Patient Saf 5:86-94
26. https://www.westgard.com/downloads/other-downloads/73-2015goal-survey-chartpack/file.html (Accesso 25 novembre 2017)

27. Lundberg GD (1981) Acting on significant laboratory results. JAMA 245:1762-1763

28. Bonini P, Plebani M, Ceriotti F et al (2002) Errors in laboratory medicine. Clin Chem 48:691-698

29. Ricós C, García-Victoria M, de la Fuente B (2004) Quality indicators and specifications for the extra-analytical phases in clinical laboratory management. Clin Chem Lab Med 42:578-582

30. Plebani M, Sciacovelli L, Aita A (2017) Quality indicators for the total testing process. Clin Lab Med 37:187-205

31. Plebani M (2017) Performance specifications for the extraanalytical phases of laboratory testing: why and how. Clin Biochem 50:550-554

32. The National Academies of Sciences, Engineering, and Medicine (2015) Improving diagnosis in health care. National Academies Press, Washington DC

33. Cappelletti P (2010) Medicina di Laboratorio. In: Plebani M, Galzigna L (eds) Trattato Italiano di Medicina di Laboratorio. Volume I Biochimica Clinica e Generale. Piccin Nuova Libraria, Padova, pp. 9-104

34. Plebani M, Laposata M, Lundberg GD (2011) The brain-to-brain loop concept for laboratory testing 40 years after its introduction. Am J Clin Pathol 136:829-833

35. Cappelletti P (2017) Brain-to-brain loop 2020: è ancora utile il ciclo di Lundberg? Riv Ital Med Lab. 13:127-133

36. Bossuyt PM, Reitsma JB, Linnet K et al (2012) Beyond diagnostic accuracy: the clinical utility of diagnostic tests. Clin Chem 58:1636-1643

37. Bossuyt PMM, McCaffery K (2009) Additional patient outcomes and pathways in evaluations of testing. In: Medical tests-white paper series. Agency for Healthcare Research and Quality (US), Rockville [Internet]. https://www.ncbi.nlm.nih.gov/ books/NBK49463/ (Accesso 25 novembre 2017)

38. Cappelletti P (2016) Appropriateness of diagnostics tests. Int J Lab Hematol 38(Suppl 1):91-99

39. Bruns DE (2001) Laboratory-related outcomes in healthcare. Clin Chem 47:1547-1552

40. Hauser RG, Shirts BH (2014) Do we now know what inappropriate laboratory utilization is? An expanded systematic review of laboratory clinical audits. Am J Clin Pathol 141:774-783

41. Graham R, Mancher M, Miller Wolman D et al (2011) Clinical practice guidelines we can trust. National Academies Press, Washington (DC)

42. Cappelletti P (2015) Linee Guida e SIPMeL. Riv Ital Med Lab 11:185-190

43. Aakre KM, Langlois MR, Watine J et al (2013) Critical review of laboratory investigations in clinical practice guidelines: proposals for the description of investigation. Clin Chem Lab Med 51:12171226

44. Cappelletti P (2016) Medicina di Laboratorio centrata sul paziente. Riv Ital Med Lab 12:193-197

45. Terzi A, Moccia F, Terzi E (2006) La sicurezza del paziente e la Medicina di Laboratorio. Il punto di vista dei cittadini. RIMeL/IJLaM 2(Suppl):42-48

46. Plebani M (2016) Towards a new paradigm in laboratory medicine: the five rights. Clin Chem Lab Med 54:1881-1891

47. Cappelletti P (2002) La SIMeL ed il futuro della Medicina di Laboratorio. Riv Med Lab-JLM 3:11-13

48. Cappelletti P (2003) La Medicina Molecolare e la Medicina di Laboratorio. Riv Med Lab-JLM 4(Suppl):19-31

49. Cappelletti P (2012) Medicina di Laboratorio e Postmodernità. Riv Ital Med Lab 8:1-15

50. Eagleman DM (2013) Why public dissemination of science matters: a manifesto. J Neurosci 33:12147-12149 